\title{
PERCEPÇÃO DE ATLETAS PROFISSIONAIS DE BASQUETEBOL SOBRE O ESTILO DE LIDERANÇA DO TÉCNICO*
}

\author{
GRAD. GEORGE DOS REIS ALBA \\ Bacharel em administração pela UCS \\ Mestrando em administração pela Universidade Federal do Rio Grande do Sul \\ (Rio Grande do Sul - Brasil) \\ E-mail: george.alba@hotmail.com \\ GRAD. TAISSON TOIGO \\ Bacharel em administração pela UCS \\ Mestrando em administração pela Universidade de Caxias do Sul (Rio Grande do Sul - Brasil) \\ E-mail: taisson@terra.com.br
}

\begin{abstract}
DR. PAULO FERNANDO PINTO BARCELLOS
Doutor em engenharia mecânica e industrial pela Marquette University (EUA) Professor adjunto da Universidade de Caxias do Sul (Rio Grande do Sul - Brasil)

E-mail: pfpbarce@ucs.br
\end{abstract}

\begin{abstract}
RESUMO
No contexto das competições esportivas, a influência do técnico pode facilitar o desempenho individual e propiciar um suporte social significativo aos demais. O estudo objetivou verificar a percepção de atletas profissionais de basquetebol masculino quanto ao estilo de liderança de seus técnicos. A pesquisa tem caráter descritivo e o instrumento de pesquisa utilizado é um questionário chamado de Escala de Liderança Revisada para o Esporte. Vinte e nove atletas de três equipes brasileiras responderam à pesquisa e os principais resultados mostram que, em duas dessas equipes, os atletas avaliam que seus técnicos utilizam, predominantemente, a autoridade pessoal para tomar as decisões com pouca ou nenhuma participação dos atletas.
\end{abstract}

PALAVRAS-CHAVE: Estilos de liderança; esportes profissionais; basquetebol.

* O presente trabalho não contou com apoio financeiro de nenhuma natureza, tampouco houve conflitos de interesses para a sua realização. 


\section{INTRODUÇÃO}

Segundo Leitão (1999), a liderança é uma das áreas da psicologia do esporte em que existe uma carência de estudos em diferentes modalidades e contextos esportivos. $\bigcirc$ autor cita que a investigação da liderança no esporte surgiu da aplicação de modelos teóricos provenientes da psicologia organizacional. No Brasil, alguns pesquisadores desenvolveram linhas de pesquisa relacionadas à liderança em atividades esportivas, tais como Samulski et al. (1998), Simões et al. (1998).

No caso específico do basquetebol, os Estados Unidos da América (EUA) mantêm uma completa hegemonia dentro do cenário competitivo mundial, motivo pelo qual a literatura científica desse país detém uma vasta quantidade de obras no que se refere à liderança no basquetebol profissional. Destacam-se: Auerbach (1977), Ramsey (1978), Weiss e Friedrichs (1986), Riley (1993), Jackson (1995) e, na última década, Kozub e Pease (200 I), Giambatista (2004), Beam et al. (2004), Loughead et al. (2006) e Frontiera (2006).

Riemer e Chelladurai (1998) referem-se em seus estudos às questões de liderança, principalmente no que diz respeito ao papel do técnico e à influência de suas ações na equipe comandada, estas estão relacionadas à mobilização, ao desenvolvimento e à utilização dos jogadores disponíveis. Assim, os atletas podem reagir positiva ou negativamente à maneira como o técnico utiliza a habilidade técnica e tática dos jogadores, seleciona e aplica estratégias de comando apropriadas, treina e instrui os atletas. A estruturação do time, ou seja, as formas como os integrantes trabalham juntos, bem como a união do grupo e as contribuições individuais e coletivas para as tarefas também podem ser consideradas aspectos importantes para a satisfação e até mesmo para um melhor desempenho dos atletas.

Nos Estados Unidos da América, segundo Frontiera (2006), é frequente a utilização da expressão "players' coach" por analistas de esporte, referindo-se aos técnicos de basquetebol que são queridos por toda sua equipe. Apenas alguns técnicos têm esse status dentro de suas equipes. Para isso eles necessitam desenvolver a confiança, motivar jogadores a um bom empenho e restabelecer a confiança após um mau desempenho.

Neste artigo, apenas a questão da liderança formal será abordada, todavia não se exclui a possibilidade de um técnico conseguir estabelecer relações de liderança com seus liderados por intermédio de características mais comuns à liderança informal, gerada espontaneamente. Esse processo se dá, segundo Covey (2002), por meio da liderança baseada em princípios e origina-se quando os valores de líder e seguidor convergem. $O$ alicerce desse tipo de liderança é a honra e ocorre quando ambos têm as mesmas finalidades ou objetivos. Um líder que busca ampliar seu 
poder baseado em princípios necessita de paciência e vontade para construir uma relação de confiança e sinceridade em longo prazo.

Verificando-se a escassez de estudos que convergem liderança aplicada a esportes coletivos e reconhecendo a importância de questões comportamentais em ações de grupo, Serpa (1993) afirmou que esse contexto indica a necessidade de se conhecer perfis de liderança que possam subsidiar novas perspectivas no processo de formação dos treinadores e que também possam melhorar a relação entre técnicos e atletas. Dado essa relevância e a não existências de trabalhos acadêmicos que tratem de liderança no basquetebol profissional brasileiro, o problema de pesquisa se configura em: Qual a percepção de atletas profissionais de basquetebol masculino quanto ao estilo de liderança de seus técnicos?

Segundo Simões (1987), o comportamento de liderança e as atitudes de um técnico junto aos membros de uma equipe representam um fator preponderante no processo da unidade e estabilidade coletiva, refletindo também no rendimento da equipe (SAMULSKI, 2002). Esse processo depende da estrutura e do tipo de comportamento de liderança do treinador diante de seus comandados e da forma como os atletas interpretam e aceitam o comportamento do técnico. A Escala de Liderança Revisada para o Esporte é um instrumento que vem sendo regularmente utilizado para verificar a percepção desses comportamentos de liderança e se encaixa com o objetivo geral do estudo, que consiste em verificar a percepção de atletas profissionais de basquetebol masculino quanto ao estilo de liderança de seus técnicos. Outro objetivo, mais específico, é identificar as correlações entre as dimensões dos estilos de liderança percebidos pelos atletas em seus técnicos.

Para Serpa (1990), as interações sociais, como as de líder e liderados, que ocorrem entre treinador e atletas devem ser consideradas como fatores determinantes no esporte profissional. Elas repercutem no comportamento e nos aspectos cognitivos e emocionais de todos os indivíduos envolvidos.

\section{FUNDAMENTAÇÃO TEÓRICA}

\section{LIDERANÇA NO ESPORTE}

Liderança pode ser definida como um processo que ocorre por meio de influências individuais dentro de um grupo para alcançar uma meta comum (Northouse, 200I). Dado que liderança está disponível a todos dentro de um grupo (LougheAD; Hardy, 2005), Carron e Hausenblas (1998) identificaram dois tipos de liderança: formal e informal. Podem ser vistos como líderes formais no esporte aqueles que foram designados pela entidade esportiva, como, por 
exemplo, o técnico. Em contraste, outros membros da equipe podem ocupar papéis de liderança informais desde que os papéis deles desenvolvam uma base de interações com os colegas.

A liderança é um dos componentes mais importantes na gestão de pessoas nas organizações, entre elas, as equipes esportivas. Liderança no esporte se refere ao processo de inspiração ou influência em atletas de uma equipe para realizar suas tarefas com entusiasmo e competência, para atender os objetivos da equipe (BRIDGes; Roquemore, 1996), e tem sido frequentemente considerada como uma das principais razões para o sucesso e o fracasso de um atleta ou uma equipe de esporte (CH'NG; KOH-TAN, 2006).

Gomes et al. (2006) afirmam que, analisando as publicações na área de liderança, se observa uma lacuna de trabalhos em contextos esportivos. Salminen e Liukkonen (1996) e Noce (2002) indicam que essa lacuna se dá principalmente relacionada a funções e ações dos treinadores. Segundo Weinberg e Gould (200 I), a atuação do líder do esporte, uma vez efetiva, contribui diretamente para um desempenho superior de todos os participantes, merecendo assim uma atenção especial da academia.

Horn ( 1 992) e Smith e Smoll (2005) indicam que as pesquisas na área de liderança no esporte se tem desenvolvido mediante pressupostos que o comportamento do líder/técnico tem um impacto significativo, de natureza negativa ou positiva, no desempenho do atleta ou no seu bem-estar psicológico. Dessa forma, a possibilidade de o treinador intervir pontualmente durante o intervalo ou no decorrer de uma partida demanda uma forte relação de confiança no atleta, o que pode apresentar-se como um dos fatores determinantes para o sucesso (SAMULSKI et al., 1998).

\section{LIDERANÇA NO BASQUETEBOL}

As trocas de técnicos na liga americana de basquetebol, a NBA (National Basketball Association), têm, diferentemente do Brasil, um ciclo pronunciado. Segundo Giambatista (2004), a maioria das mudanças de treinadores (6 I \%) acontece logo após o fim de uma temporada. Treinadores contratados imediatamente depois de uma temporada têm aproximadamente seis meses e meio para integrar e trabalhar com o time. Assim, esse período é crucial para aprendizagem e adaptação mútua de jogadores e técnico. Até porque jogos não estão sendo ganhos ou perdidos, como na temporada de campeonatos. No Brasil, o basquetebol, assim como o futebol, promove um número de trocas de técnicos considerável durante as competições.

Riemer e Chelladurai (1995) sugeriram que liderança efetiva é um fator importante na satisfação de jogadores de esportes coletivos. De fato, uma relação entre essas duas variáveis já tem certo conhecimento dentro do domínio esportivo (Riemer; Chelladural, 1995; Weiss; Friedrichs, 1986). Chelladurai (1978) incluiu a 
satisfação do atleta como uma consequência importante do comportamento do técnico no modelo multidimensional de liderança em esporte que desenvolveu.

Amorose e Horn (2000) indicaram, em estudos anteriores, que os jogadores de basquete com motivação intrínseca mais alta perceberam que seus treinadores possuem estilos de liderança que enfatizam treino e instrução. Beam et al. (2004) descobriram que um número significativo de jogadores de basquete universitário nos EUA prefere um comportamento de liderança de seus treinadores baseado em gênero, nível de competição, dependência de tarefa e variabilidade de tarefa.

Tharp e Gallimore (1976) identificaram em John Wooden, técnico de basquete dez vezes campeão nacional de basquetebol universitário pela University of Califórnia, Los Angeles (UCLA), um estilo de comportamento predominantemente autocrático, que, segundo eles, se tratava da maneira mais eficiente de liderança no basquetebol (o que fazer para fazer isso). Porém Sherman et al. (2000) encontraram em pesquisas na Austrália jogadores de basquetebol que preferiram uma realimentação mais positiva e um comportamento de treinamento democrático.

\section{ESCALA DE LIDERANÇA REVISADA PARA O ESPORTE}

Chelladurai (1978) desenvolveu o Modelo Multidimensional de Liderança (Multidimensional Model of Leadership - MML) especialmente para o ambiente esportivo. Teorias anteriores de liderança focavam apenas sobre a figura do líder, enquanto esse modelo incorporou o líder, os membros da equipe e a situação. Cada uma dessas três dimensões foi considerada igualmente importante. $O$ modelo possui três estados de comportamento de liderança: requerida, preferida e real. Como consequência da congruência desses comportamentos do líder, o desempenho do grupo e a satisfação dos membros denotam uma liderança eficaz (COSTA, 2003). A figura I representa de forma esquemática o modelo de Chelladurai (1990).

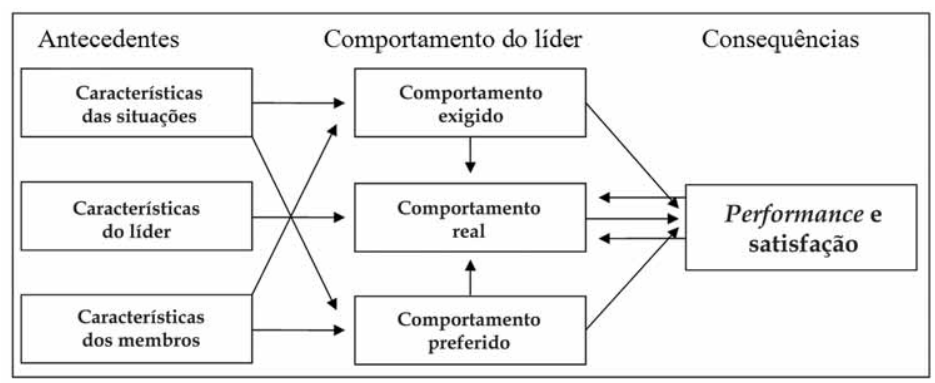

Figura I: Multidimensional Model of Leadership. Fonte: Adaptado de Chelladurai (1990, p. 330). 
Avaliando as consequências desse modelo, Chelladurai (1990), Salminen e Liukkonen (1996) e Jorge (1998) constataram, com base em resultados de pesquisas, que o rendimento ótimo e a satisfação do grupo são atingidos quando os comportamentos exigidos, reais e preferidos do líder são consistentes e congruentes.

Com o objetivo de realizar estudos sobre esse modelo, Chelladurai e Saleh ( 1980 ) desenvolveram a Escala de Liderança para o Esporte (Leadership Scale for Sport - LSS). Entretanto, após revisar pesquisas que se utilizaram da LSS, Chelladurai (1990) identificou alguns problemas na escala, tais como o enfoque dos itens ser mais voltado para as frequências do que para o contexto dos comportamentos e a escala ter sido construída baseada em testes da área organizacional sem relação direta com o esporte. Nesse sentido, Zhang et al. (1997) revisaram a LSS e a aprimoraram, desenvolvendo a Escala de Liderança Revisada para o Esporte (Revised Leadership Scale for Sports - RLSS) a partir de cinco estágios: a) adição de dimensões e itens; b) revisão linguística da versão inicial da RLSS; c) teste de validade de conteúdo; d) teste de validade de construto e consistência interna; e) proposta da versão final. As dimensões são divididas pelo estilo de decisão predominante (democrático ou autocrático) e pelo estilo de interação que o treinador adota (demais dimensões). No quadro I, as dimensões abordadas pelo questionário são brevemente contextualizadas e faz-se uma indicação da quantidade numérica de questões no instrumento.

Quadro I - Descrição das dimensões da RLSS.

\begin{tabular}{|l|l|c|}
\hline \multicolumn{1}{|c|}{ Dimensões } & \multicolumn{1}{|c|}{ Comportamentos típicos do técnico } & $\begin{array}{c}\text { Quantidade } \\
\text { de questões }\end{array}$ \\
\hline Democrática & $\begin{array}{l}\text { Permite a participação dos atletas nas decisões da equipe, respeita } \\
\text { e aceita os direitos dos jogadores. }\end{array}$ & 12 \\
\hline Autocrática & $\begin{array}{l}\text { Uso da autoridade pessoal para tomar as decisões com pouca ou } \\
\text { nenhuma participação dos atletas. }\end{array}$ & 8 \\
\hline Reforço & Reconhece e recompensa o rendimento dos jogadores. & 12 \\
\hline Situacional & $\begin{array}{l}\text { Considera as características individuais dos atletas (maturação, } \\
\text { habilidade) para nortear o trabalho. }\end{array}$ & 10 \\
\hline Treino-instrução & $\begin{array}{l}\text { Foco na melhora do rendimento do atleta por meio do planejamento } \\
\text { e estruturação das atividades. Fornece instruções técnicas e táticas. }\end{array}$ & 10 \\
\hline Suporte social & $\begin{array}{l}\text { Valorização do bem-estar dos jogadores, busca promover um clima } \\
\text { positivo nas relações interpessoais da equipe. }\end{array}$ & 8 \\
\hline
\end{tabular}

Fonte: Adaptado de Lopes (2008). 
Segundo Gil (2008), as etapas a serem seguidas na realização da pesquisa são descritas neste fragmento. A pesquisa poderá ser caracterizada em dois critérios básicos: quanto aos fins e quanto aos meios de investigação (VERGARA, 2006). Este trabalho se caracteriza por ser um estudo descritivo com abordagem quantitativa, utilizando-se de questionário com escala Likert de I (sempre) a 5 (nunca) como técnica investigativa para levantamento dos dados. Essas características do estudo foram evidenciadas por Gil (idem), ao relatar que as pesquisas descritivas têm por objetivo básico descrever as características de determinada população ou fenômeno e estabelecer possíveis relações entre variáveis.

Para Hair et al. (2005), os dados quantitativos devem ser coletados por meio de várias escalas numéricas. As abordagens quantitativas são muito utilizadas, quando se possui problema de pesquisa ou modelos teóricos bem definidos. A validação desses conceitos geralmente envolve a utilização de questionários como a RLSS. O questionário consiste em um número de questões apresentadas por escrito às pessoas, tendo como objetivo o conhecimento de opiniões, crenças, interesses, expectativas, situações vivenciadas etc.

O questionário utilizado originou-se de um construto traduzido por Chelladurai (1978), revisto por Zhang et al. ( 1997) e validado no Brasil por Lopes (2006). Esse instrumento de pesquisa apresenta seis dimensões de liderança: democrática, autocrática, reforço, situacional, treino-instrução e suporte social. Chama-se Escala de Liderança Revisada para o Esporte (Revised Leadership Scale for Sports - RLSS). Cada um dos enunciados desse questionário descreve um comportamento específico que o técnico pode manifestar. Assim, o objetivo do estudo consiste em verificar a percepção de atletas profissionais de basquetebol masculino quanto ao estilo de liderança de seus técnicos.

A RLSS é formada por sessenta questões fechadas, separadas em dois estilos de decisão e seis dimensões da liderança, conforme o quadro I. É utilizada uma escala do tipo Likert, composta de cinco pontos, e as cinco alternativas de resposta são as seguintes: sempre ( 100\%), frequentemente (75\%), ocasionalmente (50\%), raramente $(25 \%)$ e nunca (0\%).

A validação da RLSS na língua portuguesa foi realizada por Lopes (idem), com treinadores e atletas, participantes do Campeonato Brasileiro de Seleções Juvenis de Voleibol de 2004, masculino e feminino, e da Superliga de Voleibol Feminino 2004-2005. Os resultados obtidos por Lopes (idem) indicaram que todas as dimensões, com exceção do comportamento autocrático, obtiveram um índice de consistência aceitável (alfa de cronbach maior que 0,70). Apesar de a dimensão de 
comportamento autocrático não atingir os índices propostos pela literatura (PASQUALI, 1999), houve uma melhora na validade e confiabilidade em relação à mesma dimensão na LSS. Lopes (idem) garante que o instrumento é adequado para o uso no contexto esportivo no Brasil. Contudo, foi realizada uma análise da consistência interna para este estudo, utilizando novamente o coeficiente alfa de cronbach, em que valores maiores ou iguais a 0,7 foram considerados indicativos de consistência interna (NuNAlly, 1978). Esse coeficiente é utilizado quando o instrumento ou suas dimensões são constituídos por itens dispostos em escala ordinal e com um mesmo número de pontos, sendo adequado para o questionário utilizado neste artigo.

Foram contatados dez dirigentes de equipes profissionais, e apenas três manifestaram interesse em participar da pesquisa. Os questionários foram respondidos pelos atletas em um período anterior ao treino, todos ao mesmo tempo em cada equipe. Foi explicado o objetivo da pesquisa e que as identidades dos atletas e equipes permaneceriam preservadas, mesmo assim um atleta da equipe Alpha e quatro atletas da equipe Gama preferiram não responder o questionário. Participaram do estudo 29 atletas profissionais de equipes de basquetebol do Brasil: 10 atletas da equipe Alpha, I I atletas da equipe Beta e 8 atletas da equipe Gama.

Os dados foram analisados por meio de estatística descritiva, apresentando-se as frequências, médias e desvio-padrão. Também foram submetidos à análise dos coeficientes de correlação, a fim de determinar o nível e a direção das relações entre as dimensões do instrumento de pesquisa. Esses coeficientes podem variar de - I,0 a I,0, e, quanto mais distante do 0 for o coeficiente, mais forte será o nível de associação. Esses coeficientes podem ser positivos ou negativos, o que irá depender da direção da relação entre as variáveis, se diretamente ou inversamente associadas (HAR et al., 2005). O software utilizado para a análise estatística foi o Microsoft Excel 2007.

\section{APRESENTAÇÃO DOS RESULTADOS}

Com o propósito de verificar a confiabilidade da escala utilizada para medir a percepção da liderança e suas dimensões, o alfa de cronbach foi calculado. Os valores encontrados foram 0,8773 para a dimensão democrática, 0,9121 para a dimensão autocrática, 0,8788 para a dimensão reforço, 0,7036 para a dimensão situacional, 0,6902 para a dimensão treino-instrução e 0,737। para a dimensão suporte social. $\bigcirc$ alfa de cronbach total do questionário encontrado foi 0,8759. Todas as dimensões, exceto a dimensão situacional e o questionário como um todo, apresentaram um nível de confiabilidade acima de 0,7, o que indica a consistência interna do instrumento. Mesmo a dimensão situacional, que ficou abaixo dessa faixa, apresentou uma diferença pouco significativa $(0,0098)$. 
Esta pesquisa foi realizada com o objetivo de verificar a percepção de atletas profissionais de basquete sobre o estilo de liderança de seus técnicos. Para tal, foi aplicado um questionário composto por questões informativas quanto ao perfil da amostra e por questões direcionadas à verificação do estilo de liderança percebido dos técnicos.

Esse estudo apresentou as seguintes características com relação à amostra: a) 24, I 4\% têm entre I 8 e 23 anos, 55, I 7\% têm entre 23 e 28 anos, e 20,69\% têm mais de 28 anos; b) 27,57\% atuam profissionalmente a menos de 5 anos, 48,28\% atuam entre 5 e 10 anos, e 24, I4\% atuam a mais de 10 anos, entretanto nenhum atleta atua profissionalmente a mais de 16 anos; c) 51 ,72\% desempenham a função de "ala", 24, $14 \%$ desempenham a função de "armador" e 24, $14 \%$ desempenham a função de "pivô".

Numa escala Likert de um a cinco, a média é três, que significa segundo o modelo utilizado uma ocorrência ocasional, ou em $50 \%$ das vezes. Médias superiores a três, portanto, indicam uma ocorrência de um comportamento na maioria das vezes. A fim de verificar as percepções existentes sobre as seis dimensões, bem como a dispersão das respostas entre os pesquisados, foi elaborada uma tabela com as médias e os desvios-padrão em cada uma das dimensões. No que se refere às duas dimensões de estilo de decisão, constatou-se a predominância do estilo autocrático sobre o democrático nas equipes Beta e Gama, e a predominância do estilo democrático sobre o autocrático na equipe Alpha.

Em relação aos estilos de interação, verificou-se que, nas equipes em que o estilo de decisão predominante é o autocrático (Beta e Gama), se evidenciam maiores médias no estilo de interação treino-instrução e menores médias nos estilos de interação reforço e suporte social. Na equipe Alpha, em que predomina o estilo de decisão democrático, esse cenário praticamente se inverte.

Tabela I - Médias das dimensões da RLSS nas equipes Alpha, Beta e Gama

\begin{tabular}{|c|c|c|c|c|c|c|c|c|c|}
\hline Equipes & \multicolumn{3}{|c|}{ Alpha } & \multicolumn{3}{|c|}{ Beta } & \multicolumn{3}{|c|}{ Gama } \\
\hline Dimensões & $\mathrm{N}$ & $\mu$ & $\sigma$ & N & M & $\sigma$ & $N$ & $\mu$ & $\sigma$ \\
\hline Democrática (Estilo de decisão) & 10 & 3,533 & 0,819 & 11 & 2,455 & 0,968 & 8 & 2,229 & 1,110 \\
\hline Autocrática (Estilo de decisão) & 10 & 2,000 & $0,87 \mid$ & II & 3,557 & 0,933 & 8 & 3,953 & 0,916 \\
\hline Reforço (Estilo de interação) & 10 & 3,933 & 0,847 & 11 & 3,182 & 1,010 & 8 & 2,542 & ।,065 \\
\hline Situacional (Estilo de interação) & 10 & 3,630 & 0,837 & II & 3,055 & 1,074 & 8 & 2,813 & 1,284 \\
\hline Treino-instrução (Estilo de interação) & 10 & 2,870 & 0,991 & II & 3,873 & 0,847 & 8 & 3,575 & 1,240 \\
\hline Suporte social (Estilo de interação) & 10 & 3,575 & 1,065 & 11 & 2,943 & 1,118 & 8 & 2,594 & 1,422 \\
\hline
\end{tabular}

Quando se analisou a correlação existente entre as dimensões do construto, verificou-se que existem alguns coeficientes de correlação significativos. 
Entre eles, aparecem fatores de correlação positivos, ou seja, o crescimento em dada questão é diretamente associado à questão que está sendo correlacionada e a fatores de correlação negativos, ou seja, o crescimento em dada questão é inversamente associado. Neste estudo consideraram-se significativos os coeficientes de correlação maiores que 0,5 ou menores que -0,5. De acordo com Hair et al. (2005), estes podem ser considerados índices de correlação de força associativa moderada ou alta. Um dado previamente esperado e confirmado na pesquisa consiste na correlação negativa significativa entre a dimensão autocrática e democrática na mesma equipe (-0,805, -0,589 e -0,560 em Alpha, Beta e Gama respectivamente) (Tabelas 2,3 e 4 ).

Tabela 2 - Correlações das dimensões de Alpha

\begin{tabular}{|l|c|c|c|c|c|c|}
\hline \multicolumn{1}{|c|}{ Dimensões } & DEM & AUT & REF & SIT & TI & SS \\
\hline Democrática (DEM) & & $-0,805$ & $0,5 / 7$ & $-0,006$ & $-0,005$ & 0,563 \\
\hline Autocrática (AUT) & & & $-0,7 / 9$ & $-0,176$ & 0,233 & $-0,648$ \\
\hline Reforço (REF) & & & & 0,541 & $-0,012$ & $0,6 / 8$ \\
\hline Situacional (SIT) & & & & & 0,063 & 0,452 \\
\hline Treino-instrução(TI) & & & & & & 0,066 \\
\hline Suporte social (SS) & & & & & & \\
\hline
\end{tabular}

Tabela 3 - Correlações das dimensões de Beta

\begin{tabular}{|l|c|c|c|c|c|c|}
\hline \multicolumn{1}{|c|}{ Dimensões } & DEM & AUT & REF & SIT & TI & SS \\
\hline Democrática (DEM) & & $-0,589$ & 0,636 & 0,539 & 0,547 & 0,734 \\
\hline Autocrática (AUT) & & & $-0,421$ & $-0,454$ & $-0,302$ & $-0,469$ \\
\hline Reforço (REF) & & & & 0,738 & 0,289 & 0,850 \\
\hline Situacional (SIT) & & & & & 0,482 & 0,573 \\
\hline Treino-instrução(TI) & & & & & & 0,134 \\
\hline Suporte social (SS) & & & & & & \\
\hline
\end{tabular}

Tabela 4 - Correlações das dimensões de Gama

\begin{tabular}{|l|c|c|c|c|c|c|}
\hline \multicolumn{1}{|c|}{ Dimensões } & DEM & AUT & REF & SIT & TI & SS \\
\hline Democrática (DEM) & & $-0,560$ & 0,233 & 0,034 & 0,287 & 0,591 \\
\hline Autocrática (AUT) & & & $-0,018$ & 0,238 & $-0,730$ & 0,120 \\
\hline Reforço (REF) & & & & 0,591 & $-0,162$ & 0,437 \\
\hline Situacional (SIT) & & & & & $-0,424$ & 0,374 \\
\hline Treino-instrução(TI) & & & & & & $-0,269$ \\
\hline Suporte social (SS) & & & & & & \\
\hline
\end{tabular}

Analisando os resultados obtidos na equipe Alpha (Tabela 2), constatou-se uma correlação negativa entre uso da autoridade pessoal para tomar as decisões com pouca ou nenhuma participação dos atletas (dimensão autocrática) e o reconhecimento e recompensa do rendimento dos jogadores (dimensão reforço) 
(correlação $=-0,7 \mid 9)$. Também, foi negativa a correlação da dimensão autocrática com a valorização do bem-estar dos jogadores, buscando promover um clima positivo nas relações interpessoais da equipe (dimensão suporte social) (correlação $=-0,648$ ). Observou-se também uma correlação positiva significativa entre as dimensões reforço e situacional (correlação $=0,54 \mathrm{I}$ ) e entre as dimensões reforço e suporte social (correlação $=0,6 \mid 8$ ).

Quanto aos resultados da equipe Beta (Tabela 3), verificou-se correlações positivas entre a participação dos atletas nas decisões da equipe (dimensão democrática) e o reconhecimento e recompensa do rendimento dos jogadores (dimensão reforço) (correlação $=0,636$ ), e, também, daquela dimensão com a valorização do bem-estar dos jogadores, buscando promover um clima positivo nas relações interpessoais da equipe (dimensão suporte social) (correlação $=0,734$ ). A dimensão autocrática também apresentou correlações positivas com a dimensão situacional (correlação = 0,539) e com a dimensão treino-instrução (correlação = 0,547). Assim, como na equipe Alpha, sinalizou-se uma correlação positiva entre as dimensões reforço e suporte social (correlação $=0,850$ ).

Já a equipe Gama (Tabela 4) apresentou resultados que denotam uma correlação negativa entre as dimensões autocrática e foco na melhora do rendimento do atleta por meio do planejamento e estruturação das atividades (dimensão treino-instrução). Reportaram-se também correlações positivas entre a dimensão democrática e suporte social (correlação $=0,59$ I) e entre a dimensão reforço e situacional (correlação $=0,59$ ) .

\section{DISCUSSÕES E CONSIDERAÇÕES FINAIS}

Ainda que algumas discussões já tenham sido discorridas durante a apresentação dos resultados, existem reflexões sobre o estudo que ainda necessitam ser abordadas devido à amplitude desse tema. As pesquisas sobre liderança no esporte, conforme Gomes et al. (2006), têm estudado a relação entre as características dos atletas e o estilo adotado pelos técnicos, quanto às consequências do estilo de liderança sobre os jogadores, como satisfação e coesão.

Os resultados encontrados nas equipes Beta e Gama quanto à intensidade da dimensão autocrática coincidem com a intensidade encontrada nos estudos de Costa (2006) nos treinadores de futebol brasileiros. Os resultados encontrados ilustram como os jogadores veem seu técnico, todavia essas informações não são suficientes para delimitar eficiência operacional ou satisfação dos atletas. A forte predominância do estilo autocrático em duas das três equipes nos remete às afirmaç̧ões de Henschen e Statler (2002), que chamam a atenção para os cuidados que os técnicos devem 
ter com o seu estilo de liderança, pois a liderança autoritária parece aumentar a probabilidade de burnout mais do que os outros estilos. Para Becker Júnior (2002), o autoritarismo pode ser uma expressão real das necessidades do técnico e será mais eficiente quando essas necessidades forem supridas.

Observou-se, também, dentro da avaliação de jogadores de uma mesma equipe, uma disparidade de algumas respostas entre os respondentes acerca de algumas dimensões. Por exemplo, enquanto houve atletas que avaliaram o técnico com uma média abaixo de dois em determinada dimensão, outros atletas o avaliaram com uma média superior a quatro. Por isso e devido à amostra do estudo presente, é prematuro sugerir prescrições definitivas relativas ao estilo de liderança de técnicos de basquetebol. Porém esta pesquisa permite avaliar a percepção de um atributo importante dentro de um contexto específico de uma atividade que, segundo Eys et al. (2007), possui alto nível de influência da liderança no comportamento do profissional, devido à alta interatividade que o ambiente esportivo proporciona.

Outra importante consideração trata-se de que os comportamentos de liderança dentro de equipes esportivas não devem limitar-se apenas à figura do técnico, visto que cada membro da equipe (comissão técnica e jogadores) pode apresentar sinais de liderança (BednareK et al., 1976; Kozub; PeAse, 200 I). Assim, a liderança pode ser encarada como uma função necessária a outros membros da equipe além do técnico (WHEELAN; JOHNSTON, 1996). Portanto, para se ter uma compreensão completa de liderança no esporte, é importante, também, analisarse a liderança que atletas, dirigentes e outros membros da equipe exercem uns sobre os outros, visto que técnicos e líderes informais normalmente diferem em comportamentos de liderança (LougheAD; HARDY, 2005). Exemplificando, Loughead e Hardy (idem) verificaram que líderes informais costumam apresentar maiores escores nas dimensões autocráticas e treinamento-instrução do que os técnicos.

Apesar das limitações desta pesquisa, acredita-se que os resultados obtidos apresentaram importantes contribuições sobre o estudo dos comportamentos de liderança em esportes profissionais. A exigência por resultados satisfatórios no esporte tem gerado investimentos significativos, tanto em questões estruturais, quanto em equipamentos e instalações modernas, como em termos de pessoal. Então, é fundamental que o técnico acompanhe essa tendência, a fim de capacitar-se para melhor administrar o grande número de variáveis presentes na atividade esportiva. 


\section{Percepción de jugadores profesionales de baloncesto sobre el estilo de liderazgo del técnico}

RESUMEN: En el contexto de las competiciones deportivas, la influencia del técnico puede facilitar el desempeño individual y propiciar un soporte social significativo a los demás. El estudio objetivó verificar la percepción de los atletas profesionales de baloncesto masculino cuanto al estilo de liderazgo de sus técnicos. La pesquisa tiene carácter descriptivo y cuantitativo y el instrumento de pesquisa utilizado es un cuestionario llamado "Escala de Liderança Revisada para o Esporte". Veinte y nueve atletas de tres equipos brasileños respondieron a la pesquisa y los principales resultados muestran que en dos equipos estudiados los atletas evalúan que sus técnicos utilizan, predominantemente, la autoridad personal para la toma de decisiones con poca o ninguna participación de ellos.

PALABRAS CLAVES: Estilos de liderazgo; deportes profesionales; baloncesto.

\section{Professional basketball players perception over their head coach's leadership style}

ABSTRACT: In the context of sports competition, the head coach's influence can facilitate individual performance and propitiate a significant social support to others. The study aims to verify the perception of male basketball professional players over their head coaches' leadership style. The research is a descriptive and quantitative one. The research instrument is a survey - Revised Leadership Scale for Sport (RLSS). Twenty nine players of three Brazilian teams answered the survey and the main results show that the athletes of two studied teams evaluate their head coaches' behavior as predominantly autocratic, using their authority to make decisions with few or without athletes participation.

KEY WORDS: Leadership styles; professional sports; basketball.

\section{REFERÊNCIAS}

AMOROSE, A. J.; HORN T. S. Intrinsic motivation: relationship with collegiate athletes' gender, scholarship status, and perceptions of their coaches' behavior. Journal of Sport and Exercise Psychology, v. 22 ( I), p. 63-84, 2000.

AUERBACH, R. Red Auerbach: an autobiography. With Joe Fitzgerald. New York: Putnam's Sons, 1977.

BEAM, J. W.; SERWATKA, T. S.; WILSON, W. J. Preferred leadership of NCAA Division I and Division II intercollegiate student-athletes. Journal of Sport Behavior, v. 27, p. I - 17, 2004.

BECKER JÚNIOR, B. Psicologia aplicada ao treinador esportivo. Novo Hamburgo: Feevale, 2002. 
BEDNAREK, F.; BENSON, L.; MUSTAFA, H. Identifying peer leadership in small work groups. Small Group Behavior, v. 7, p. 307-316, 1976.

BRIDGES, F. J.; ROQUEMORE, L. L. Management for athletic/sport administration: theory and practice. 2. ed. Decatur (GA): ESM Books, 1996.

CARRON, A. V.; HAUSENBLAS, H. A. Group dynamics in sport. 2. ed. Morgantown (W): Fitness Information Technology, 1998.

CHELLADURAI, P. A contingency model of leadership in athletics. Unpublished Doctoral Dissertation - Department of Management Sciences, University of Waterloo, Canada, 1978. . Leadership in sports: a review. International Journal of Sport Psychology, n. 21, 1990.

.; SALEH, S. Dimensions of behavior in sports: development of leadership scale. Journal of Sport Psychology. n. 2, p. 34-45, 1980.

CH'NG, A.; KOH-TAN, A. Managing sport: concepts and issues of non-profit organizations. Singapore: Prentice Hall, 2006.

COSTA, I. T. Análise de perfil de liderança de treinadores de futebol do Campeonato Brasileiro Série A/2005. Dissertação (Mestrado) - Escola de Educação Física, Fisioterapia e Terapia Educacional, Universidade Federal de Minas Gerais, Belo Horizonte, 2006.

COSTA, V. T. Análise do perfil de liderança atual e ideal de treinadores de futsal de alto rendimento, através da escala de liderança do desporto (ELD). Dissertação (Mestrado) - Escola de Educação Física, Fisioterapia e Terapia Ocupacional, Universidade Federal de Minas Gerais, Belo Horizonte, 2003.

COVEY, S. R. Liderança baseada em princípios. Rio de Janeiro: Campus, 2002.

EYS, M. A.; LOUGHEAD, T. M.; HARDY, J. Athlete leadership dispersion and satisfaction in interactive sport teams. Psychology of Sport and Exercise, v. 8, p. 28I-296, 2007.

FRONTIERA, J. The relationship between leadership, efficacy belief, and performance among boys' high school basketball players. Thesis (Doctoral) - School of Physical Education, West Virginia University. Morgantown (WW), 2006.

GIAMBATISTA, R. C. Jumping through hoops: a longitudinal study of leader life cycles in the NBA. The Leadership Quarterly, v. I5, p. 607-624, 2004.

GIL, A. C. Métodos e técnicas de pesquisa social. 6. ed. São Paulo: Atlas, 2008.

GOMES, A. R.; SOUSA, S. A.; CRUZ, J. F. Charismatic, transformational and visionary dimensions in sport leadership: toward news paths for the study of coach-athletes relationships. In: HUBER, N. S.; HARVEY, M. (Eds.). Leadership at the crossroads. College Park: University of Maryland, The James MacGregor Burns Academy of Leadership, 2006. p. 84-94. 
HAIR, Jr. J.; MONEY, A.; SAMOUEL, P.; BABIN, B. Fundamentos de métodos de pesquisa em administração. Porto Alegre: Bookman, 2005.

HENSCHEN, K.P., STALTER, T. O burnout e staleness atlético: uma saga constante. In: BECKER JÚNIOR, B. (Org.). Psicologia aplicada ao treinador esportivo. Novo Hamburgo: Feevale, 2002. p. 187-202.

HORN, T. Leadership effectiveness in the sport domain. In: HORN, T. (Ed.). Advances in sport psychology. Champaign (IL): Human Kinetics, 1992. p. I8I-199.

JACKSON, P. Sacred hoops. New York: Hyperion, 1995.

JORGE, P. Aplicação transcultural da escala de liderança no desporto na ginástica rítmica desportiva. Dissertação (Mestrado) - Faculdade de Motricidade Humana, Universidade Técnica de Lisboa, Lisboa, 1998.

KOZUB, S. A.; PEASE, D. G. Coach and player leadership in high school basketball. Journal of Sport Pedagogy: Teaching and Coaching in Sport, v. 7, p. I- 15, 2001 .

LEITÃO, J. A relação treinador-atleta: percepção dos comportamentos de liderança e de coesão em equipes de futebol. Tese (Doutorado) - Faculdade de Ciências do Desporto e Educação Física, Universidade Coimbra, Porto, 1999.

LOPES, L. S. Avaliação do comportamento tático no futebol de campo: um estudo do perfil de tomada de decisão do atleta e sua relação coma percepção sobre o estilo de liderança do treinador. Dissertação (Mestrado) - Escola de Educação Física, Universidade de Brasília, Brasília, 2008.

LOPES, M. A relação do estilo de liderança dos treinadores de voleibol com a satisfação das atletas na Superliga Feminina 2004-2005. Dissertação (Mestrado) - Escola de Educação Física, Fisioterapia e Terapia Educacional, Universidade Federal de Minas Gerais, Belo Horizonte, 2006.

LOUGHEAD, T. M.; HARDY, J. An examination of coach and peer leader behaviors in sport. Psychology of Sport and Exercise, v. 6, p. 303-312, 2005.

.; HARDY, J.; EYS, M. A. The nature of athlete leadership. Journal of Sport Behavior, v. 29, p. |42-158, 2006.

NOCE, F. Liderança. In: SAMULSKI, D. Psicologia do esporte: manual para a educação física, fisioterapia e psicologia. São Paulo: Manole, 2002. p. 219-248.

NORTHOUSE, P. G. Leadership: theory and practice. 2. ed. Thousand Oaks (CA): Sage, 200 I. NUNNALLY, J. C. Psychometric theory. New York: McGraw-Hill, 1978.

PASQUALI, L. Instrumentos psicológicos: manual prático de elaboração. Brasília: LabPAM, 1999. 
RAMSEY, J. The coach's art. With John Strawn. Forest Grove: Timber Press, 1978.

RIEMER, H. A.; CHELLADURAI, P. Leadership and satisfaction in athletics. Journal of Sport and Exercise Psychology. v. 17, p. 276-293, 1995.

Development of the Athlete Satisfaction Questionnaire (ASQ). Journal of Sport and Exercise Psychology, v. 20, p. 127- 156, 1998.

RILEY, P. The winner within. New York: Putnam's Sons, 1993.

SALMINEN, S.; LIUKKONEN, J. Coach-athlete relationship and coaching behavior in training sessions. International Journal of Sport Psycology, v. 27, p. 59-67, 1996.

SAMULSKI, D. Psicologia do esporte: manual para a educação física, psicologia e fisioterapia. Barueri: Manole, 2002.

.; NOCE, F; PUSSIELDI, G. Estudo comparativo dos estilos de liderança entre treinadores de voleibol e natação. In: SILAMI-GARCIA, E.; LEMOS, K.; GRECO, P. (Orgs.). Temas atuais em educação física e esportes III. Belo Horizonte: Editora UFMG, 1998. p. I39154.

SERPA, S. O treinador como líder: panorama actual da investigação. Ludens, v. I2, p. 23-32, 1990.

Avaliação dos processos de interação treinador-atleta e liderança no desporto. Ludens, v. 13, n. I, p. 9-16, 1993.

SHERMAN, C. A.; FULLER, R.; SPEED, H. D. Gender comparisons of preferred coaching behaviors in Australian sports. Journal of Sport Behavior, v. 23 (4), p. 389-406, 2000.

SIMÕES, A. Estudo do comportamento de liderança dos técnicos de handebol. Dissertação (Mestrado) - Escola de Educação Física, Universidade de São Paulo, São Paulo, 1987.

.; RODRIGUES, A.; CARVALHO, D. Liderança e as forças que impulsionam a conduta de técnico e atletas de futebol, em convívio grupal. Revista Paulista de Educação Física, São Paulo, n. 12, v. 2, p. 134-|44, 1998.

SMITH, R. E.; SMOLL, F. L. Assessing psychosocial outcomes in coach training programs. In: ACKFORT, D.; DUDA, J. L.; LIDOR, R. (Eds.). Handbook of research in applied sport and exercise psychology: international perspectives. Virgínia: Fitness Information Technology, 2005. p. 293-316.

THARP, R. G.; GALLIMORE, R. What a coach can teach a teacher. Psychology Today, v. 9, p. 75-78, 1976.

VERGARA, S. C. Projetos e relatórios de pesquisa em administração. 7. ed. São Paulo: Atlas, 2006. 
WEINBERG, R. S.; GOULD, D. Fundamentos da psicologia do esporte e do exercício. 2. ed. Porto Alegre: Artmed. 2001.

WEISS, M. R.; FRIEDRICHS, W. D. The influence of leader behaviors, coach attributes, and institutional variables on performance and satisfaction of collegiate basketball teams. Journal of Sport Psychology, v. 8, p. 332-346, 1986.

WHEELAN, S. A.; JOHNSTON, F. The role of informal member leaders in a system containing formal leaders. Small Group Research, v. 27, p. 33-55, 1996.

ZHANG, J.; JENSEN, B. E.; MANN, B. L. Modification and revision of the leadership scale for sport. Journal of Sport Behavior, v. 20, n. I, p. 105-122, 1997.

Recebido: 12 set. 2009

Aprovado: 31 mar. 2010

Endereço para correspondência:

George dos Reis Alba

Rua Alcides Maya, 174 - Bairro Cinquentenário

Caxias do Sul-RS

CEP $95012-020$ 